being used to do this and to extract the fragments from the bladder. In this case the mucous membrane of the prostatic urethra certainly came away. The tissue left between the cavity and the rectum was very thin. There was a good deal of hæmorrhage for a short time after removal of the fingers from the bladder. This was washed out with sterilised water, the ends of the wound were closed with sutures and a large drainage-tube was inserted right down to the bottom of the cavity formerly occupied by the tumour. I was unable to replace the four portions of the growth in their correct relations or to trace the former position of the urethra. The weight was six and a half ounces and the size was that of a large orange.

The operation was over at 4.40 P.M., but the subsequent course of the case was not all plain sailing by any means. Within an hour a severe rigor set in. The temperature at 6 P.M. was $101.4^{\circ}$ and at 9 P.M. $103^{\circ}$, the pulse being 133 and the respirations 40 . At $10 \mathrm{P} . M$. it had begun to abate : the temperature had dropped to $101 \cdot 2^{\circ}$. That night the patient slept for two and a quarter hours and took milk and brandy. On the next dav his temperature at $6 \mathrm{~A} . \mathrm{M}$. was $99^{\circ}$ and at 6 P. M. it was $100 \cdot 4^{\circ}$. Never after this did it exceed $100^{\circ}$, but the pulse continued to range from 92 to 104 and the respirations from 20 to 28 for many days. On the third and fourth days his condition caused me some anxiety on account of heart weakness, and he was injected with one-sixtieth of a grain of strychnine sulphate every quarter of an hour, but on the fifth day he could eat a baked custard. On the seventh day a number of large blotches of a purpuric eruption came out on the backs of both hands and lasted about a week. gradually fading away. I am at a loss to account for this. On the ninth day the patient stated that he passed a few drops of urine through the penis, but as his bed was always wet it was impossible either to confirm or to deny his statement. Up to this time the wound had been dressed with pads of absorbent gauze and wool, but as the fat had become very sloughy the dressing was changed on the tenth day to boric acid fomentations. On the eleventh day the patient sat up in a chair for a short time. The wound having become encrusted with phosphates I tried the effect of soaking the lint in a solution (1 in 20) of acetic acid for a few days but without good results. On the thirteenth day he was sufficiently well to dine off chicken, custard, and fruit. Un the twenty-sixth day he passed altogether 15 ounces of urine naturally and on the twenty eighth day Mr. Bacon passed a soft rubber catheter to assist him. The leakage from the wound gradually ceased and the urine passed more and more through the penis until, on the fifty-seventh day, a collodion dressing was applied. A few days before this the bladder had been washed out daily through a catheter and this attention was required now and again afterwards until he was finally sent home on the seventy-fourth day. He is now able to walk about and to retain or to pass his urine as he wirhes. He says he feels better than he has for years.

Southampton.

\section{A CASE OF ACUTE EPENDYMITIS IN AN INFANT.}

By J. A. COUTTS, M.B. Cantab., F.R.C.P. Lond., PHYSICIAN TO THE EAST LONDON HOSPITAL FOR CHLLDREN.

CASES of primary acute ependymitis leading to the formation of pus in the cerebral ventricles are of such exceptional rarity as to merit that each one should be put on record. Apart from the result of the bursting of a cerebral abscess into their cavities, or as a consequence of suppuration in the sac of a spina bifida, the presence of unmistakeable pus in the cerebral ventricles is a rare occurrence under any conditions. Very occasionally pus in the ventricles is found post mortem in cases of purulent meningitis. Far most frequently, however, the turbid fluid found in even the acutest of these last cases affords no naked-eye appearance of pus, whatever evidence of its purulent character may be forthcoming from micro-copical or chemical examinations. The interest in the present case lies in the fact that large masses of pus were found in the ventricles post mortem, without atsy other lesions being discovered in the meninges or cerebral substance.

Euendymitis as a distinct pathological condition is more frequently dealt with by American a riters than by those in this country. References to it, both as an acute and chroni- cundition, may be tound in the works of Delafield and Prudden, Osler, Emmett Holt, and others in America and by Sir William Gowers amongst English authorities. As far as I can gather all these writers treat acute ependymitis from a purely pathological standpoint and merely as a condition discoverable post mortem in connexion with disease of other parts of the brain or its membranes. I know of no instance where an attempt has been made by any writer to connect any symptoms observable during life with the ventricular conditions discovered post mortem. As regards the symptoms arising from the chronic form of ependymitis we perhaps stand on a less uncertain basis. More than 20 years ago, in a graduation thesis at Cambridge, I combated the view that chronic hydrocephalus had any causal origin in rickets and ventured the opinion that possibly an explanation of the fcrmer complaint would be found in a primary chronic inflammatory condition of the ventricular ependyma. This view is not inconsistent with the conditions discoverable post mortem and, even if far from satisfactorily established, is, I believe, one very commonly held at the present day.

The difficulty in dealing with the acuter forms of ependymitis lies in the fact that almost invariably the ventricular affection is complicated with other gross lesions of the brain or its membranes and it becomes a matter of impossibility to assign to each separate part its due share in the production of symptoms. In my present case, in the later stages at least, the acute ventricular inflammation was probably the sole disorder and it is no unfair assumption that some of the later symptoms observable during life were directly ascribable to it. As these symptoms, however, were in no sense distinctive or characteristic and varied but little from day to day, I have thought it well briefly to summarise them from the excellent and copious notes of my former house physician, Dr. G. A. Finlayson, now bacteriologist at Singapore.

The patient, a female infant, aged three months, was admitted into the East London Hospital for Children under my care on Nov. 19th, 1902. In the family history of the case there was nothing special to note. The father and mother and two remaining children were healthy. Two children were dead; one had died from pneumonia with measles and the other was found dead in bed. There had been no miscarriages. The patient was a breast.fed infant and had been fairly healthy until the onset of the present illness. This occurred two days before her admission to hospital with diarrhoea and vomiting. The stools, three or four daily, were green and not offensive. The vomiting occurred soon after taking the breast. Early in the morning of the day of admission the infant had a convulsive attack, the arms and legs were held rigid for some time, then twitching occurred in the extremities. The attack lasted about 15 minutes and soon after the child was brought to the hospital. Just before the onset of the fit she seemed to have pain in the abdomen with drawing up of her legs and screaming.

On admission the patient was found to be a small, pale, and rather badly nourished infant. The eyes were bright and the pupils were equal and convergent strabismus was strongly marked. The breathing was irregular both in $\mathrm{xhythm}$ and in depth. The pulse varied extremely in force and in rhythm, becoming faster when the breathing was quicker and very slow and almost intermittent when a period of apnoea supervened. There was no definite convulsive attack, but the infant wa; restless and had irregular spasmodic tremors of the hands. The legs were drawn up on the abdomen and were slightly flexed. Kernig's sign was not present. The knee-jerks were very active-they were brisk and were excited by a very slight tap on the tendon. There was slight retraction of the head but no marked rigidity of the post-cervical muscles. The anterior fontanelle was tense and bulging. The sagittal and frontal sutures were widely open. There was nothing calling for special note in the examination of the chest. The skin was elastic. The tongue was furred. The abdomen was so't and distended. The infant could not take the breast and had to be fed nasally. The temperature on admission was $103^{\circ} \mathrm{F}$. but it fell to $101^{\circ}$ in the course of a few hours.

The symptoms varied but little till the death of the infant on Dec. 29th and can be summarised as follows. The vomiting persisted until the end-three or four times a day-generally soon after being fed. There was also slight obstinate diarrhœea which continued in spite of treatment until a week before death. After a fortnight in hospital the fits recurred and after this there were few days in which there were not 
several convulsive attacks. At first these consisted of clonic spasmodic contractions of the left arm and leg and both the mouth and eyes were drawn to the left side. The later attacks were general in character and showed no unilateral tendency. Each fit lasted a considerable time and was ushered in by a shrill scream on the part of the infant. Towards the end they became very frequent. The temperature, which had been $103^{\circ}$ on admission, presented from the beginning a marked up-and-down type. At first the maxima attained seldom went much above $101^{\circ}$ and on several occasions the temperature dropped temporarily below the normal. Later, when the fits became more numerous, the temperature assumed a higher range and on one or two occasions it went up beyond $104^{\circ}$. The knee-jerks continued exceedingly brisk and a slight tap on the patella tendon would throw the limb into a state of clonic spasm with as many as from 10 to 20 contractions. The head retraction became more marked and the post-cervical muscles more rigid, and towards the end there was considerable opisthotonos. The anterior fontanelle continued very tense and bulging. The day before death an attempt was made to relieve the cerebral tension by drawing off fluid from the ventricles by way of the anterior fontanelle, but this failed owing doubtlessly to the eye of the needle getting blocked by one of the masses of lymph or pus. For the first three weeks the infant remained fairly plump, but before death emaciation became extreme. The total duration of the illness was exactly six weeks.

The following are the notes of the post-mortem examination 18 hours after death, made by Dr. Leonard S. Dudgeon, the pathologist to the hospital :-

The child was very emaciated. No skin lesions were to be seen or felt. No spina bifida was detected. The pharynx, trachea, lungs, pleura, mediastinal glands, heart, pericardium, and the thymus gland were all found to be healthy to the naked eye. The pulmonary artery was opened in situ, but there was no evidence of any antemortem clot. The ahdominal cavity and its contents, the liver, spleen, kidney, suprarenals, pancreas, stomach, intestines, and glands were all found to be normal. Head.-All the cranial sinuses were wermal. There were no abnormal changes in any of the bones of normal. There were no abnormal changes in any of the bones of branes. - No middle ear or mastoid disease was noted. Cerebral membranes. - Nothing abnormal was seen to suggest any past or present hæmorrhage or any new growths were seen in any part of the brain hæmorrhage or any new growths were seen in any part of the brain
substance. There was extreme bulging at the base of the brain in the region of the optic commissure. As soon as the third ventricle was opened a large quantity of thick turbid fluid poured out and also large flakes of lymph and pus. All the ventricles were extremely dilated and contained similar fluid and pus. At the level of the foramen of Magendie there appeared to be complete blocking. There was no dilatation of the central canal of the spinal cord to the naked eye There was no inflammation of the spinal membranes in the cervical region-in fact, the cord and membranes appeared to be quite normal in this region. An examination of the spinal cord in the dorsal and lumbar region could not be made, but no fluid could be drawn off from the lumbar region of the cord. The fluid from the ventricle was not offensive. The pus was very thick, whitish-green, and very fibrinous. The dilatation of the lateral ventricles was so extreme that the brain tissue only formed a very thin outer wall to the cavities of the ventricles which were extremely dilated in their whole course. There was an extremely thick inflammatory deposit attached to the ependyma of the ventricles which could be scraped off, leaving a rough surface beneath with great dilatation of the veins coursing along the walls of the ventricles. Also a few scattered pinhead hæmorrhages were seen in the walls of the lateral ventricles. Bacteriological examination of the pus from the ventricular cavities.-Cover-slip preparations of the pus showed large numbers of cocei arranged without exception in the form of long chains. Cultures were made on most of the ordinary Inedia. In every case small colonies grew similar in every respect to those of members of the streptococci family. Cover-slip preparations of the pus were made from the growths on the various media and in every specimen examined cocei in the form of very long chains were the was no capsule to be seen to the cocci from the original specimen of the pus. No inoculation experiments were mare with the organism. the pus. No inoculation experiments were marle with the
There was no cause discovered for the ventricular infection.

Needless to say the conditions discovered in the ventricles were totally unsuspected before the necropsy. The close similarity of the symptoms to those of meningitis is sufficiently obvious, and during life the case was naturally taken to be one of the post.basic variety of that complaint. That the diagnosis was wrong, as least as regards the variety of any meningitis that may have been temporarily present, is proved by the bacteriological examination, for the organism found was assuredly not the one so commonly associated with the narne of Dr. G. F. Still. Whether the streptococcus discovered was a variety of the pneumococcus Dr. Dudgeon prefers to leave unsettled from the data at his disposal. Under this supposition as to the nature of the micro-organism it is, of course, possible that an early pneumococcal meningitis, leading to blocking of the basal foramina, may have at one time existed and cleared up before death. For such an assumption, however, there was absolutely no evidence forthcoming at the post-mortem examination. A more natural and more justifiable conclusion in the circumstances is that, rare as the condition may be, the present case was really one of a primary acute inflammation of the ventricular ependyma. Such an inflammation and its results would be thoroughly compatible with the effects discovered post mortem, and possibly with the symptoms observable during life. As has been said, these symptoms were in the main those of a post-basal meningitis. On the severity and time of appearance of one or two of the symptoms some stress may, perhaps, be laid as lending aid in distinguishing between the two conditions. In the present case the onset was more acute and the fits were much more frequent and persistent than in an ordinary case of post-basic meningitis. The temperature, too, towards the end of the third week began to rise, whilst in most cases of post-basic meningitis it would by that time have reached the normal or fallen below it. One other point seems worthy of some special notice. In a sick infant aged three months only the degree of consciousness present is hard to estimate. The fact, however, that from the moment of her admission to the hospital the infant had lost the power of sucking and had to be fed nasally is strong presumptive evidence that the amount of consciousness appropriate to the age of the child was already in entire abeyance. So early a loss of consciousness, in less than two days from the onset of the illness, is a rare occurrence in any form of meningitis. On the failure to elicit Kernig's sign I would personally place but little weight The inability to draw off fluid from the ventricle, where one was fairly certain the point of the needle was within that cavity, ought, perhaps, to have led to the conclusion that the case was not an ordinary one of post-basic meningitis, but deductions drawn from such a procedure hardly enter into the domains of practical diagnosis.

The main features of acute ependymitis in infants, as far as one is justified in attempting to settle them from a single case, would seem to be acute onset with vomiting, a persistent temperature of the hectic type rising towards the end of the illness, very early loss of consciousness, very frequent fits dating almost from the onset, and early and persistent bulging of the anterior fontanelle, along with convergent strabismus. I much fear, however, that at the present time the differential diagnosis between such cases and those of meningitis is practically impossible, especially when it is remembered how widely cases of the last disorder vary as regards the prominence and times of appearance of their numerous symptoms.

In conclusion, I have to acknowledge my indebtedness to Dr. Dudgeon, not only for the care which he bestowed on the post-mortem and bacteriological examinations, but for several references to the literature of acute ependymitis that I should otherwise have missed.

Upper Berkeley-street, w.

\section{A CASE OF DROPSY, THE RESULT OF INFLAMMATION OF THE KIDNEYS, TREATED BY LAPAROTOMY.}

\section{BY HENRY PAUL PLANER, M.D.}

IN the following article I wish to draw the attention of my fellow physicians to a case of dropsy resulting from inflammation of the kidneys, completely relieved by laparotomy and the treatment following the operation-a case of so much interest that it seems to me to be worthy of publication.

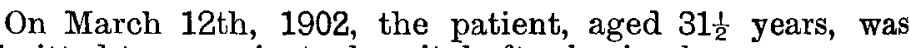
admitted to my private hospital after having been unsuccessfully tapped at another hospital, from which she was dismissed as incurable on March 7th, 1902, with the prognosis of her speedy decease. Her condition on admission was as follows. She was very anæmic and had almost a bilious complexion; the lungs were healthy, the heart was not enlarged, and the heart-beats were clear. The liver reached far beyond the right arch of the ribs and was very much enlarged. The spleen was three fingers' breadth beyond the usual limit and painful to touch. The kidneys, according to external percussion and palpation, were enlarged and sensitive to touch. The uterus was somewhat small and moderately retroverted. Menstruation 